The publisher regrets that the articles in Machine Learning, volume 62, issue 1-2, incorrectly listed Springer as the copyright holder. The following articles' copyright holders are the author(s):

\title{
Introduction to Special Issue
}

Hendrik Blockeel, David Jensen, Stefan Kramer

\section{PRL: A Probablistic Relational Language}

Lise Getoor, John Grant

\section{Propositionaization-Based Relational Subgroup Discovery with RSD}

Nada Lavrač, Filip Železný

Distribution-based Aggregation for Relational Learning with Identifier Attributes Claudia Perlich, Foster Provost

\section{Markov Logic Networks}

Matthew Richardson, Pedro Domingos

XRules: An Effective Algorithm for Structural Classification of XML Data

Mohammad J. Zaki, Charu C. Aggarwal 